\title{
Percutaneous Transhepatic Biodegradable Biliary Stent Placement for Benign Biliary Strictures
}

\author{
Shaima Abulqasim, MBBS ${ }^{1,2}$ Mohammad Arabi, MD, FRCP, FPAIRS, FSIR ${ }^{1,2}$ Khalid Almasar, MBBS 1,2 \\ Bayan AlBdah, Statistician II ${ }^{2,3}$ Refaat Salman, EBIR, MHBA ${ }^{1,2}$
}

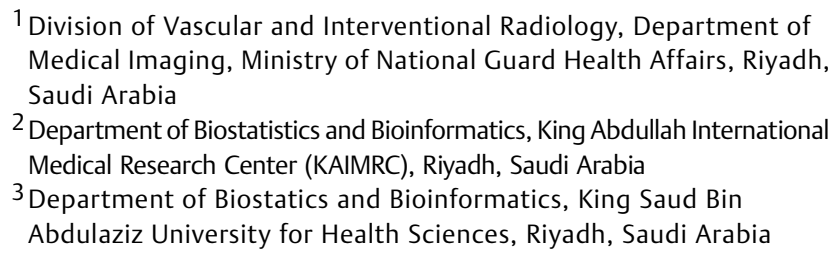
Medical Research Center (KAIMRC), Riyadh, Saudi Arabia

Abdulaziz University for Health Sciences, Riyadh, Saudi Arabia

Address for correspondence Refaat Salman, EBIR, MHBA, King Abdulaziz Medical City and King Abdullah Specialized Children's Hospital, Ministry of National Guard Health Affairs, Riyadh, Saudi Arabia (e-mail: Salmanre@ngha.med.sa; refaat.salman@gmail.com).

Dig Dis Interv 2021;5:307-310.

\begin{abstract}
This article aimed to assess the safety and effectiveness of biodegradable stents in the management of benign biliary strictures. This is a retrospective observational study that included all adult patients who had biodegradable stent placement for a benign cause of biliary stricture between July 2016 and August 2019. Nineteen patients were included. Seventeen patients had liver transplant. One patient had hepaticojejunostomy due to primary sclerosing cholangitis and one patient had iatrogenic left main bile duct occlusion. Stents were successfully deployed in all 19 patients (technical success:

Keywords

- benign biliary stricture

- biliary stent

- biodegradable

- percutaneous transhepatic cholangiopathy $100 \%)$. Patency rate was $90 \%(17 / 19)$ at 6 months and $80 \%(12 / 15)$ at 12 months. Seven patients in the study had stricture recurrence and needed reintervention with mean time to reintervention of 418 days (range: $8-1,155$ days). There was one major complication due to cholangitis and sepsis, which required a treatment course with piperacillin/tazobactam for 10 days. No procedure-related pancreatitis or deaths occurred. Biodegradable stents are a safe and effective treatment option for benign biliary strictures and can achieve long-term patency without the need for reinterventions.
\end{abstract}

Benign biliary strictures are usually iatrogenic. ${ }^{1,2}$ These strictures can be difficult to treat and cause significant morbidity and health care cost due to the need for repeated interventions.

The use of biodegradable biliary stents (BBS) is a relatively new treatment option for these strictures. It is thought to have comparable patency to endoscopic treatment and plastic stent insertion, with less need for reintervention and recurrent admissions. ${ }^{2-4}$

This study is a continuation of a previous article published in $2019,{ }^{5}$ with a larger number of patients and longer followup duration.

received

March 20, 2021

Issue Theme Special Communication

accepted after revision

June 24, 2021

\section{Materials and Methods}

This is a single-center retrospective observational study conducted in a tertiary hospital. Institutional review board approval was obtained. The study included all adult patients who had benign biliary strictures treated using BBS between July 2016 and August 2019. This study is a continuation of the article by Arabi et al, ${ }^{5}$ with a larger number of patients, longer follow-up duration, and additional cause of benign stricture (inadvertently clipped left duct during cholecystectomy). The current study includes 19 patients, of which 12 (c) 2021. Thieme. All rights reserved. Thieme Medical Publishers, Inc., 333 Seventh Avenue, 18th Floor, New York, NY 10001, USA
DOI https://doi.org/ 10.1055/s-0041-1733796. ISSN 2472-8721. 
Table 1 Stricture features

\begin{tabular}{|c|c|c|c|c|}
\hline \multirow[b]{2}{*}{ Level of stricture } & \multicolumn{2}{|c|}{ Total } & \multicolumn{2}{|c|}{ Reintervention } \\
\hline & $N$ & Percent & $\begin{array}{l}\text { No } \\
(n=12)\end{array}$ & $\begin{array}{l}\text { Yes } \\
(n=7)\end{array}$ \\
\hline Hepaticojejunal & 4 & 21.05 & $\begin{array}{l}2 \\
16.67\end{array}$ & $\begin{array}{l}2 \\
28.57\end{array}$ \\
\hline Choledochocholedocal & 14 & 73.68 & $\begin{array}{l}9 \\
75.00\end{array}$ & $\begin{array}{l}5 \\
71.43\end{array}$ \\
\hline Left main bile duct & 1 & 5.26 & $\begin{array}{l}1 \\
8.33\end{array}$ & $\begin{array}{l}0 \\
0.00\end{array}$ \\
\hline \multicolumn{5}{|l|}{ Etiology of stricture } \\
\hline $\begin{array}{l}\text { Anastomotic stricture } \\
\text { post-liver transplant }\end{array}$ & 16 & 84.21 & $\begin{array}{l}10 \\
83.33\end{array}$ & $\begin{array}{l}6 \\
85.71\end{array}$ \\
\hline $\begin{array}{l}\text { primary sclerosing } \\
\text { cholangitis (PSC) }\end{array}$ & 2 & 10.53 & $\begin{array}{l}1 \\
8.33\end{array}$ & $\begin{array}{l}1 \\
14.29\end{array}$ \\
\hline $\begin{array}{l}\text { latrogenic bile duct } \\
\text { occlusion during } \\
\text { cholecystectomy }\end{array}$ & 1 & 5.26 & $\begin{array}{l}1 \\
8.33\end{array}$ & $\begin{array}{l}0 \\
0.00\end{array}$ \\
\hline
\end{tabular}

were included in the previous cohort. Patients with malignant stricture were excluded.

The related laboratory information, demographic data, and imaging data were reviewed. Baseline bilirubin (total and direct), aspartate aminotransferase, alanine aminotransferase, and alkaline phosphatase were obtained for all patients and then used for a follow-up in conjunction with imaging.

A total of 20 patients who underwent BBS insertion were identified; one patient died after 2 months due to progressive hepatic failure and was excluded. Nineteen patients were included in the study (15 males, $79 \%$ ) with a mean age of 55 years (range: $23-72$ years).

Seventeen patients had liver transplant (15 choledochocholedochal anastomosis and 2 hepaticojejunal anastomosis; - Table 1, - Fig. 1). One patient had hepaticojejunostomy due to primary sclerosing cholangitis and one patient had iatrogenic left main bile duct occlusion, which was inadvertently closed by surgical clips during cholecystectomy (-Fig. 2).

The mean time since surgery was 40.8 months (range: 1.8-199 months). All patients had previous sessions of percutaneous transhepatic balloon cholangioplasty with an average of three sessions (range: 1-6). Patients received preprocedural antibiotics with $1 \mathrm{~g}$ cefazolin IV as per protocol. The BBS used was (ELLA-CS, s.r.o., Hradec Kralove, Czech Republic) a self-expandable bare stent made of biodegradable material (polydioxanone). The placement was done according to the instruction for use from the manufacturer (the technique was described in the previous article). ${ }^{5}$

Clinical success was evaluated using laboratories, imaging, and being tube-free post BBS placement. Technical success was defined as correct deployment of the stent and documenting its patency immediately postdeployment. Endpoints were either last follow-up (at least 6 months) or reintervention (endoscopic, percutaneous, or surgical). Time
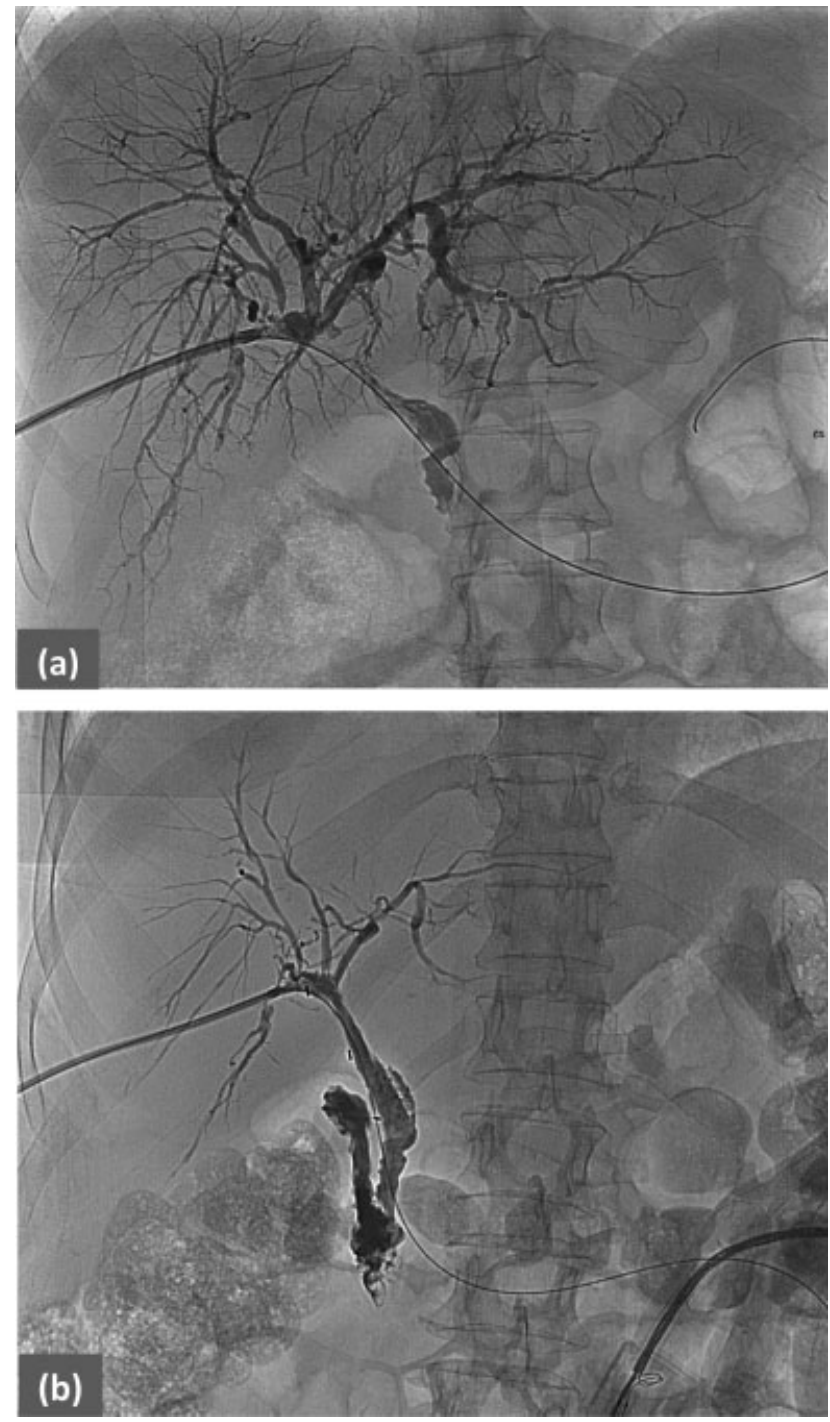

Fig. 1 Intraprocedure images from a patient who presented with jaundice and abnormal liver function test 10 months after liver transplant. She underwent four sessions of cholangioplasty with recurrent anastomotic stricture. (a) Initial cholangiogram shows highgrade anastomotic stricture. (b) Cholangiogram after deployment of BBS shows patent stent with contrast flow to the duodenum. On follow-up for the next 3 years, the patient remained tube-free with normalized liver functions.

to reintervention was defined as the time interval between initial stent placement and any subsequent intervention (endoscopic or percutaneous) done due to stricture recurrence (diagnosed by laboratory and/or imaging evidence).

Complications were evaluated and classified according to the new adverse event classification published by the Society of Interventional Radiology Standards of Practice Committee. ${ }^{6}$

\section{Results}

Stents were successfully deployed in all 19 patients (technical success: $100 \%)$. The median follow-up time was 468 days (range: 8-1,211 days). 

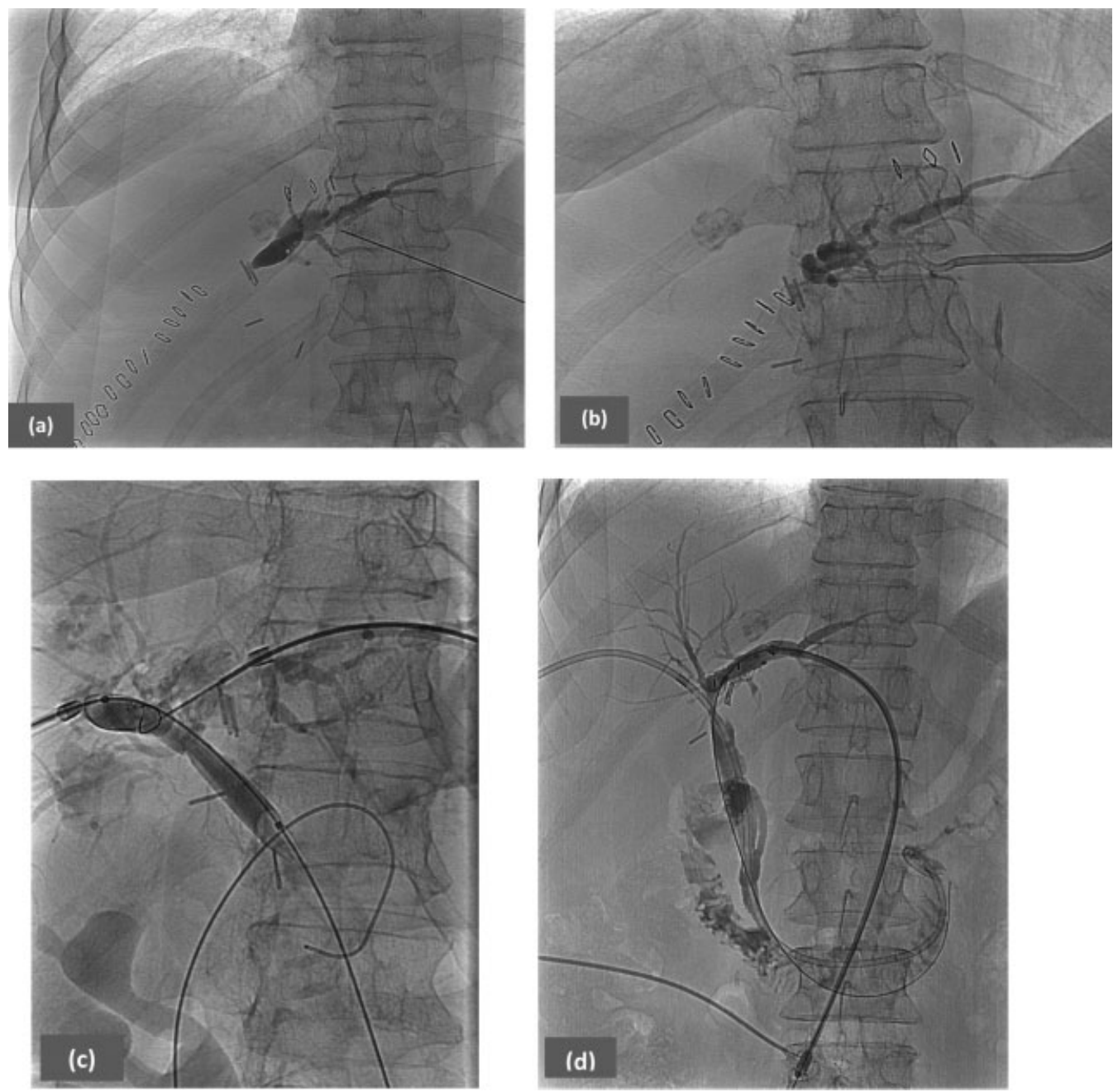

Fig. 2 A 72-year-old female with history of cholecystectomy complicated by iatrogenic left bile duct occlusion. (a, b) Cholangiograms showing abrupt cutoff of the left main bile duct. Three surgical clips are noted at the site of occlusion. Initial trial to cross the occlusion was not successful; so, an 8-Fr external biliary drain was inserted. (c) The patient was brought later for recanalization. The right-side bile ducts were accessed and a balloon was inserted and then targeted by a needle from the left side. (d) The area of stricture was predilated and then $8 \mathrm{~mm} \times 4 \mathrm{~cm}$ BBS was placed. Cholangiogram shows patent stent with contrast flow to the duodenum.

Twelve patients (63.2\%) remained tube-free during the follow-up and had improved laboratory results from baseline and did not need further intervention at median follow-up time of 650 days (range: 168-1,333 days). Patency rate was $90 \%(17 / 19)$ at 6 months and $80 \%$ $(12 / 15)$ at 12 months without the need for additional interventions.

Seven patients (36.8\%) in the study had stricture recurrence and needed reintervention with median time to reintervention of 418 days (range: 8-1,155 days; - Table 2).

The total procedure-related complication rate was $21 \%$ (4/19). Three patients had minor complications (one to two spikes of fever with negative blood cultures; no further intervention or prolonged management was needed). One patient had major complications presented with fever and positive blood culture with Escherichia coli diagnosed with cholangitis and sepsis that required a treatment course with piperacillin/tazobactam for 10 days. The same patient had stent migration to the duodenum noticed in follow-up tube cholangiogram after 5 days of insertion but did not need further intervention. No procedure-related pancreatitis or deaths occurred.

Patients who did not need reinterventions after BBS placement were noted to have higher rate of prior sessions of intervention with balloon cholangioplasty prior to stenting (median: 3, range: 1-6). While those who required

Table 2 Reintervention types

\begin{tabular}{|l|l|}
\hline Type of reintervention & Frequency \\
\hline Balloon cholangioplasty alone & 1 \\
\hline $\begin{array}{l}\text { Balloon cholangioplasty } \\
\text { and internal-external biliary drain }\end{array}$ & 2 \\
\hline $\begin{array}{l}\text { Endoscopic retrograde } \\
\text { cholangiopancreatography (ERCP) } \\
\text { with insertion of plastic stent }\end{array}$ & 2 \\
\hline External biliary drain & 1 \\
\hline Internal-external biliary drain & 1 \\
\hline
\end{tabular}


reinterventions were noted to have lower number of prior interventions (median: 2 , range: $1-3$ ).

\section{Discussion}

Biodegradable stents have been used to treat esophageal strictures with promising results. It is also being studied for airways and intestinal and colonic strictures caused by various benign and malignant causes. ${ }^{7-9}$

Biodegradable stents are a novel treatment option for benign biliary strictures that obviate the need for stent removal. Studies have shown low migration rate, less need for reintervention, and acceptable patency. ${ }^{3,10}$

In a retrospective multicenter study by Mauri et al, 107 patients with refractory benign biliary strictures treated using BBS were reviewed. Technical success was $98 \%$ without major complications. The study suggested that percutaneous placement of BBS is a safe and feasible procedure for the management of refractory biliary stricture, with estimated stricture recurrence rate of $7.2,26.4$, and $29.4 \%$ at 1,2 , and 3 years, respectively. The estimated mean time to stricture recurrence was 38 months. ${ }^{4}$

Battiste et al reported the outcome of BBS stents use to treat refractory benign biliary anastomotic strictures postliver transplantation. Success rate of complete resolution of anastomotic stricture was achieved in $72 \%$ of patients after a median follow-up time of 27 months. Only one complication of hemobilia was observed. ${ }^{10}$

In a recent systematic review and meta-analysis reported by Almeida and Donato comparing BBS versus multiple plastic stents (MPS), three studies regarding BBS and six regarding MPS were included. The overall success rate was comparable (83\% for BBS, $84 \%$ for MPS). Reintervention was less with BBS. However, cholangitis and hemobilia postprocedure were higher with BBS (cholangitis: $24.1 \%$ with BBS vs. $6.1 \%$ with MPS; hemobilia: $3 \%$ with BBS vs. $<1 \%$ with MPS). ${ }^{2}$

A larger prospective, multicenter, observational, nonrandomized study (the BiELLA study) was conducted in 11 tertiary hospitals in Spain and included 150 patients to study safety and efficacy of BBS in the treatment of benign biliary strictures. The primary mean patency rates for stent were $86.7,79.6$, and $78.9 \%$ at 12,36 , and 60 months, respectively. Biliary restenosis and occlusion occurred in 40 patients (26.6\%). ${ }^{11}$

In our study, results show that BBS stents are feasible and a relatively safe method for the treatment of benign biliary strictures, with one reported major complication.

Success rate was slightly lower than in the previously mentioned studies, with $63 \%$ resolution of strictures and improved biochemical markers with the patient being free from tube dependency. A third of patients needed reintervention after mean follow-up time of 13.9 months. These patients were found to have a smaller number of previous interventions (median: 2, range: $1-3$ ) prior to stent place- ment compared with the group who did not require reintervention (median: 3, range: $2-6$ ). This suggests that BSS can be helpful in patients who require multiple cholangioplasty to reduce the need for further interventions.

Limitations of the study include its small sample size that is related to the infrequent procedure, the retrospective nature of the study, and lack of comparison group.

The use of biodegradable stents is a safe and effective treatment option for benign biliary strictures and can help obviate the need for repeated interventions and long-term tube-dependency. Additional studies with a larger sample size are suggested to confirm these results and further assess the cost-effectiveness and impact on quality of life compared with other methods of treatment.

Conflict of Interest

None declared.

\section{References}

1 Kapoor BS, Mauri G, Lorenz JM. Management of biliary strictures: state-of-the-art review. Radiology 2018;289(03):590-603

2 Almeida GG, Donato P. Biodegradable versus multiple plastic stent implantation in benign biliary strictures: a systematic review and meta-analysis. Eur J Radiol 2020;125:108899

3 Siiki A, Sand J, Laukkarinen J. A systematic review of biodegradable biliary stents: promising biocompatibility without stent removal. Eur J Gastroenterol Hepatol 2018;30(08):813-818

4 Mauri G, Michelozzi C, Melchiorre F, et al. Benign biliary strictures refractory to standard bilioplasty treated using polydoxanone biodegradable biliary stents: retrospective multicentric data analysis on 107 patients. Eur Radiol 2016;26(11):4057-4063

5 Arabi M, Alrehaili B, Salman R, et al. Percutaneous transhepatic biodegradable stent placement for benign anastomotic biliary strictures: short-term outcomes of a single-institution experience. Arab J Intervent Radiol 2019;3:19-22

6 Khalilzadeh O, Baerlocher MO, Shyn PB, et al. Proposal of a new adverse event classification by the Society of Interventional Radiology Standards of Practice Committee. J Vasc Interv Radiol 2017;28(10):1432-1437.e3 [erratum in J Vasc Interv Radiol 2018;29(1):146]

7 Fuccio L, Hassan C, Frazzoni L, Miglio R, Repici A. Clinical outcomes following stent placement in refractory benign esophageal stricture: a systematic review and meta-analysis. Endoscopy 2016;48 (02):141-148

8 Lorenzo-Zúñiga V, Moreno-de-Vega V, Marín I, Boix J. Biodegradable stents in gastrointestinal endoscopy. World J Gastroenterol 2014;20(09):2212-2217

9 Stehlik L, Hytych V, Letackova J, Kubena P, Vasakova M. Biodegradable polydioxanone stents in the treatment of adult patients with tracheal narrowing. BMC Pulm Med 2015;15:164

10 Battistel M, Senzolo M, Ferrarese A, et al. Biodegradable biliary stents for percutaneous treatment of post-liver transplantation refractory benign biliary anastomotic strictures. Cardiovasc Intervent Radiol 2020;43(05):749-755

11 De Gregorio MA, Criado E, Guirola JA, et al; Spanish Group BiELLA (SERVEI) Absorbable stents for treatment of benign biliary strictures: long-term follow-up in the prospective Spanish registry. Eur Radiol 2020;30(08):4486-4495 